\title{
FUZZY WEAK LINK APPROACH TO THE TWO-STAGE DEA
}

\author{
Dimitris Despotis ${ }^{1}$ AND Dorota Kuchta ${ }^{2, *}$
}

\begin{abstract}
This paper refers to a recent approach to two-stage DEA called the weak link approach. It underlines the lack of solution uniqueness in this approach to DEA and the fact that in order for the solution to the weak link approach to be unique, the decision maker needs to express a preference on which Pareto solution would be most satisfactory. In this paper, we propose to use a fuzzy set approach called fuzzy bicriterial programming to help the decision maker to express this preference. Fuzzy bicriterial programming is explained and then applied to the weak link approach to the DEA. It is shown that for each candidate (Pareto) solution to the original weak link approach, there exists an expert opinion that can lead to the unequivocal selection of this solution due to the use of the fuzzy approach. The proposal is illustrated with examples.
\end{abstract}

Mathematics Subject Classification. 90C70, 90B50.

Received October 9, 2016. Accepted September 18, 2019.

\section{INTRODUCTION}

Decision envelopment analysis (DEA) deals with the problem of assessing the efficiencies of a group of decision making units (DMUs), each of which transforms a set of inputs into a set of outputs. Recently, a great deal of research has been devoted to multi-stage DEA (as opposed to the single-stage case), in which each DMU is a chain of several transformations of inputs into outputs, and the outputs of the previous stage may become the inputs for the next stage. Two-stage DEA is a special case of multi-stage DEA (e.g. [3-5]). Much research has also been devoted to the application of fuzzy approaches to single- or multi-stage DEA).

There are basically two general approaches to the two-stage (and generally multi-stage) DEA problem: the decomposition approach and the composition approach $([2,4])$.

The decomposition approach (represented by the additive and multiplicative approach) initially calculates the overall efficiency of each DMU and only then decomposes this overall efficiency into the efficiencies of the individual stages. The decomposition process is not unequivocal and may lead to various individual stage efficiencies. In the decomposition approach, the only efficiency the decision maker controls is the overall efficiency; the stage efficiencies are not clearly defined and are therefore difficult to assess and control $[2,4,5]$. This makes it difficult to introduce any improvements or to control them: such activities have to be performed at the level of individual stages. Furthermore, the overall efficiency of the DMU in the decomposition approach is arbitrarily

Keywords. Two-stage DEA, weak link approach, fuzzy preferences, fuzzy multicriteria programming.

1 University of Piraeus, School of Information and Communication Technologies, Pireus, Greece.

2 Wroclaw University of Science and Technology, Faculty of Computer Science and Management, Wroclaw, Poland.

*Corresponding author: dorota.kuchta@pwr.edu.pl 
defined either as the sum (in the additive approach) or the multiplication (in the multiplicative approach) of the efficiencies of individual stages, which is rather limiting and in many cases contrary to intuition (e.g. it is not clear why the efficiency of the chain of supply and production processes should be defined as a sum or a product of the individual efficiencies of the supply process and the production process).

In the composition approach, the starting point is the assessment of the individual stage efficiencies. These are individually assessed and controlled, and only later composed into an overall efficiency of both stages, while the definition of the latter can be chosen by the decision maker. A representative example of the composition approach is the weak link approach, proposed by Despotis et al. [4], in which the overall efficiency of the DMU is defined as the minimum of the efficiencies of both stages. This approach "enables the decision maker to identify the stage which causes the inefficiency of the system, and to effectively improve the performance of the system" [8] and is line with more general approaches to supply chain and project management based on bottlenecks and the Goldratt theory of constraints (e.g. [16]). It gives concrete indications as to what needs to be improved in the concrete stages of individual DMUs.

Thus, the basic difference between the decomposition approach and the composition approach to two-stage DEA is that:

(a) In the decomposition approach, the overall efficiency of both stages is determined in the first instance. Its definition (with respect to the unknown individual efficiencies of the individual stages) is fixed and cannot be changed by the decision maker, although it is not always intuitively obvious. It is decomposed into the individual efficiencies of the individual stages only in the second step, and this decomposition is neither unequivocal nor controlled by the decision maker.

(b) The composition approach consists of two steps. In the first step, the individual efficiencies of both stages are determined, with full control by the decision maker. It is only in the second step that the overall efficiency of the DMU is determined, and this is fully controlled by the decision maker. In the composition approach referred to in this paper, called the weak link approach, the definition of the overall efficiency of both stages seems to be intuitive and supported by the well-known theory of constraints. Hence, this approach was chosen as the subject of the present paper.

The weak link approach has one requirement. It selects one of the Pareto optimal solutions, and the solution will only be unique if the decision maker determines the search orientation, enabling an unequivocal selection of one of the Pareto optimal solutions on the Pareto frontier. Thus far, two specific search orientations have been proposed in the literature $[4,5]$ but a general framework for a procedure that would allow an arbitrary search orientation selection is still absent. A fuzzy approach may offer a solution to this problem.

Hence, the objective of this paper is to propose a novel fuzzy application of the weak link approach to the DEA [4] that can define a generalisation of the weak link approach proposed in the literature thus far.

The reader is reminded that fuzzy sets have two basic interpretations [20]:

(A) They may represent incomplete knowledge (e.g. we can state by means of fuzzy sets that a certain magnitude will be about five, but we do not know yet the exact value). This interpretation is used in all the papers listed above, which apply a fuzzy approach to DEA (where fuzziness represents the incompletely known inputs, outputs, weights etc.);

(B) They may represent the preferences of the decision maker (e.g. we can say that we will accept only values greater than five, but we will be completely happy only with values greater than seven). To our knowledge, this approach has not yet been applied to DEA, and particularly to multi-stage DEA. We therefore aim to fill this gap in the current knowledge.

The prefer ences of the decision maker, expressed by means of fuzzy numbers, indicate a unique search direction and for each DMU will permit a unique solution of the two-stage DEA problem to be chosen from among the Pareto optimal ones.

The structure of the paper is as follows. In Section 2, we give basic information about the two-stage DEA and the weak link approach, which is based on bicriterial mathematical programming problems. In Section 3, 
we present fuzzy sets as a tool for defining preferences and fuzzy bicriterial programming. In Section 4, fuzzy modelling and programming will be related to the weak link approach to two-stage DEA. Section 5 presents conclusions and further research perspectives.

\section{Two-stage DEA: Basic Definitions}

Suppose we have $n$ DMUs, $\mathrm{DMU}_{k}, k=1, \ldots, K$.

In two-stage DEA, each $\mathrm{DMU}_{k}, \quad k=1, \ldots, K$ transforms inputs into outputs two times, in two stages. We assume here that the outputs of the first stage are the inputs for the second stage. In more general cases, it is possible to use more general models, e.g. to let the second stage take additional, external inputs.

The notation here is based on that in [5], and is illustrated by the following figure.

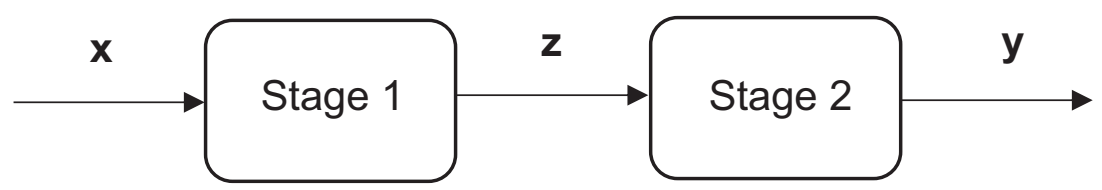

Figure 1. General scheme for the two-stage process with no external inputs in the second stage [5].

The notation is as follows: the inputs to the first stage are denoted as $x_{i}^{k}, i=1, \ldots, I$; the outputs of the first stage, which are also the inputs for the second stage, are denoted as $z_{l}^{k}, l=1, \ldots, L$; and the outputs of the second stage are denoted as $y_{j}^{k}, j=1, \ldots, J$.

In two-stage DEA, each fixed $k_{0}$ th DMU $\left(k_{0} \in\{1,2, \ldots K\}\right)$ can choose the weights of all the inputs and outputs in the two-stage process to present itself in the best light, while the weights for $y_{j}^{k_{0}}, j=1, \ldots, J$ (which are both the outputs of the first stage and the inputs of the second stage) must be the same in the first and second stages.

In the two-stage process, we assume that the output-based approach, "showing itself in the best light" means maximising two objective functions at the same time ([4]), i.e. solving the following bicriteria problem:

$$
\begin{aligned}
& \frac{\sum_{l=1}^{L} t_{l}^{k_{0}} z_{l}^{k_{0}}}{\sum_{i=1}^{I} w_{i}^{k_{0}} x_{i}^{k_{0}}} \rightarrow \max \\
& \frac{\sum_{j=1}^{J} v_{j}^{k_{0}} y_{j}^{k_{0}}}{\sum_{l=1}^{L} t_{l}^{k_{0}} z_{l}^{k_{0}}} \rightarrow \max \\
& \frac{\sum_{l=1}^{L} t_{l}^{k_{0}} z_{l}^{k}}{\sum_{i=1}^{I} w_{i}^{k_{0}} x_{i}^{k}} \leq 1, k \in\{1,2, \ldots K\} \backslash\left\{k_{0}\right\} \\
& \frac{\sum_{j=1}^{J} v_{j}^{k_{0}} y_{j}^{k}}{\sum_{l=1}^{L} t_{l}^{k_{0}} z_{l}^{k}} \leq 1, k \in\{1,2, \ldots K\} \backslash\left\{k_{0}\right\}
\end{aligned}
$$

where $t_{l}^{k_{0}}, v_{j}^{k_{0}}, w_{i}^{k_{0}} \geq 0, i=1, \ldots, I, j=1, \ldots, J, l=1, \ldots, L$ are the decision variables.

In the above model, $w_{i}^{k_{0}}, t_{l}^{k_{0}}, v_{j}^{k_{0}}, l=1, \ldots, L$ are the weights selected for the $\mathrm{DMU}_{k_{0}}$ that shows it "in the best light". 
Once we have obtained the solution from the above model, the question arises as to the overall efficiency of the two-stage process. Before we answer this question, we separate the two stages, giving two relative efficiencies for the given $\mathrm{DMU}_{k_{0}}$ : that of the first stage and that of the second stage.

$$
\begin{aligned}
\text { Efficiency of the first stage: } \vartheta_{k_{0}}=\frac{\sum_{l=1}^{L} \stackrel{k_{0}}{t} z_{l}^{k_{0}}}{\sum_{i=1}^{I} \underset{-i}{k_{0}} x_{i}^{k_{0}}} \\
\text { Efficiency of the second stage: } \xi_{k_{0}}=\frac{\sum_{j=1}^{J} \stackrel{k_{0}}{\stackrel{-}{*} y_{j}^{k_{0}}}}{\sum_{l=1}^{L} \underset{-l}{t}{ }_{-l}^{k_{0}} z_{l}^{k_{0}}}
\end{aligned}
$$

where $\vartheta_{k_{0}}, \xi_{k_{0}}$ are the "optimal" values of the objective functions (2.2) and (2.3), respectively, in a solution to the model in (2.1), and $w_{-i}^{k_{0}}, t_{-l}^{k_{0}}, v_{-j}^{k_{0}}$ are the corresponding values of the decision variables in the "optimal" solution of (2.1).

As mentioned above, in the weak link approach, which is a composition approach to the two-stage DEA problem, the efficiencies $\vartheta_{k_{0}}, \xi_{k_{0}}$ are determined in the first step, before the overall efficiency of the $\mathrm{DMU}_{k_{0}}$. The overall efficiency of $\mathrm{DMU}_{k_{0}}, e_{k_{0}}$ is then defined as the "weak link", as follows:

$$
e_{k_{0}}=\min \left\{\vartheta_{k_{0}}, \xi_{k_{0}}\right\}
$$

In the weak link approach, we do not define or consider the notion of efficient or inefficient units. The value of $e_{k_{0}}$ in equation (2.4) makes it possible to rank the decision units from the most to the least efficient. The least efficient ones may be then required to improve, and this will start with the weak link, i.e. the least efficient stage. The value of equation (2.4) serves mainly to rank the DMUs. The lowest ranked units undergo a deeper analysis in which both the efficiencies in (2.2) and (2.3) are analysed and, if possible, improved.

$\vartheta_{k_{0}}, \xi_{k_{0}}$ are selected from the Pareto optimal solutions to (2.1). This approach means that the solution to (2.1) may be different depending on the Pareto solution that is selected. In most cases, the set of Pareto solutions is infinite. The decision maker needs to give some information on the search direction within the solution space of (2.1) in order to make the choice of the solution unequivocal. This freedom of choice from among several Pareto solutions is in line with the philosophy of the DEA approach: the decision maker can influence the image of the DMU. However, in the two-stage case, the decision maker must find a compromise solution and select the efficiencies of both the weak link and the second stage from among the available Pareto solutions, in the knowledge that improving one of these necessarily worsens the other.

Despotis et al. $[4,5]$ suggest starting with the information about the so-called ideal solution, i.e. the solution that is composed of the optimal solutions of the following one-criteria problems, referring separately to each of the two stages:

$$
\begin{aligned}
& \frac{\sum_{l=1}^{L} t_{l}^{k_{0}} z_{l}^{k_{0}}}{\sum_{i=1}^{I} w_{i}^{k_{0}} x_{i}^{k_{0}}} \rightarrow \max \\
& \frac{\sum_{l=1}^{L} t_{l}^{k_{0}} z_{l}^{k}}{\sum_{i=1}^{I} w_{i}^{k_{0}} x_{i}^{k}} \leq 1, k \in\{1,2, \ldots K\} \backslash\left\{k_{0}\right\}
\end{aligned}
$$

where $t_{l}^{k_{0}}, w_{i}^{k_{0}} \geq 0, \quad i=1, \ldots, I, j=1, \ldots, J, l=1, \ldots, L$ are the decision variables. 
The optimal value of the objective function in (2.5) is denoted as $T_{k_{0}}$. This represents the relative efficiency of $\mathrm{DMU}_{k_{0}}$ in the first stage.

$$
\begin{aligned}
& \frac{\sum_{j=1}^{J} v_{j}^{k_{0}} y_{j}^{k_{0}}}{\sum_{l=1}^{L} t_{l}^{k_{0}} z_{l}^{k_{0}}} \rightarrow \max \\
& \frac{\sum_{j=1}^{J} v_{j}^{k_{0}} y_{j}^{k}}{\sum_{l=1}^{L} t_{l}^{k_{0}} z_{l}^{k}} \leq 1, k \in\{1,2, \ldots K\} \backslash\left\{k_{0}\right\}
\end{aligned}
$$

where $t_{l}^{k_{0}}, v_{j}^{k_{0}} \geq 0, \quad i=1, \ldots, I, j=1, \ldots, J, l=1, \ldots, L$ are the decision variables.

The optimal value of the objective function in (2.6) is denoted as $P_{k_{0}}$, and this corresponds to the relative efficiency of $\mathrm{DMU}_{k_{0}}$ in the second stage.

In order to find a unique solution to (2.1), in the papers by Despotis et al. $[4,5]$, two approaches were assumed, both of which refer the final solution of (2.1) to the ideal point $\left(T_{k_{0}}, P_{k_{0}}\right)$. The following approaches are proposed:

(i) Despotis et al. [4] assume that the solution to (2.1) must fulfil the condition that the ratio $\vartheta_{k_{0}} / \xi_{k_{0}}$ is equal to the ratio $T_{k_{0}} / P_{k_{0}}$. Thus, the relationship between the ideal points is maintained if both stages are considered as a single system;

(ii) Despotis et al. [5] consider the solution to (2.1) to be the point $\left(\vartheta_{k_{0}}, \xi_{k_{0}}\right)$, which is the closest point to the ideal point $\left(T_{k_{0}}, P_{k_{0}}\right)$ in the sense of the weighted Tchebycheff norm $\left(L_{\infty}\right.$ norm).

Despotis et al. [4] mention that points other than those indicated in options (i) and (ii) above can be also determined. However, no definitive indications are given as to how to make the final decision on which point $\left(\vartheta_{k_{0}}, \xi_{k_{0}}\right)$ should be chosen by the decision maker. The fuzzy approach that is proposed in the later part of this paper can help the decision maker to express his or her preferences in a formal way.

It should be underlined that the solution to (2.1) or any of its one-criterion versions can be found by means of solving linear programming problems, although problem (2.1) is not linear. This is done by applying the $\mathrm{C}-\mathrm{C}$ transformation and the bisection search.

We will illustrate the weak link approach by means of Example 1.

\section{Example 1}

In this example, we have 30 DMUs. We will concentrate here on the 18th one. Finding all the Pareto solutions for the problem in (2.1) formulated for $n_{o}=18$ gives the following Pareto set (the curve AE) (Tab. 1).

In Figure 1, point $F$ represents the ideal point $\left(T_{18}, P_{18}\right)=(0.5046,1)$. The feasible set for problem $(2.1)$ for $k_{0}=18$ is formed from the curve $\mathrm{AE}(\mathrm{A}:(0.2378,1), \mathrm{E}:(0.5046,0.4910))$ and the orthogonal projections of points $\mathrm{A}$ and $\mathrm{E}$ on the axes together with the respective axes sections. From Figure 1, we can see all the Pareto solutions to problem (2.1) for $k_{0}=18$, and thus all the candidates for the final solution to the two-stage DEA. Figure 1 also shows the two Pareto solutions to problem (2.1) for $k_{0}=18$, obtained under the two assumptions mentioned above:

(i) point $\mathrm{C}=\left(\vartheta_{18}, \xi_{18}\right)=(0.3536,0.7007)$ is a solution under the assumption that the ratio $\vartheta_{18} / \xi_{18}$ is equal to the ratio $T_{18} / P_{18}$; we then have a final efficiency for $\mathrm{DMU}_{18}$ of $e_{18}=0.3536$;

(ii) point $\mathrm{B}=\left(\vartheta_{18}, \xi_{18}\right)=(0.3082,0.8037)$ is a solution under the assumption that the distance between the ideal point and $\mathrm{C}$ (in the sense of the weighted Tchebycheff norm $\left(L_{\infty}\right.$ norm)) should be minimised, $e_{18}=0.3082$.

Any other point on the Pareto frontier could also be chosen, for example point D $(0.468,0.528)$, (which will be interpreted later on) or even points A and E; however, the decision maker must express his/her preferences in order to make the final decision. The present application of the weak link approach allows only the selection 
TABLE 1. Entry data for Example 1 [4].

\begin{tabular}{lllllll}
\hline \hline $\mathrm{DMU}_{k}$ & $x_{1}^{k}$ & $x_{2}^{k}$ & $z_{1}^{k}$ & $z_{2}^{k}$ & $y_{1}^{k}$ & $y_{2}^{k}$ \\
\hline 1 & 69.5 & 68.6 & 56.6 & 84.4 & 48.7 & 62.8 \\
2 & 40.2 & 66.2 & 88 & 47.2 & 85.8 & 28.3 \\
3 & 81.3 & 89.8 & 44.4 & 18.4 & 38.3 & 20.7 \\
4 & 55 & 97.9 & 28.7 & 41.6 & 38.2 & 10.3 \\
5 & 56.2 & 59.1 & 26.5 & 52.7 & 44.2 & 17.4 \\
6 & 64.8 & 64.4 & 14.7 & 70.5 & 86.6 & 22.9 \\
7 & 79.2 & 68.1 & 63.5 & 39.3 & 47.6 & 35 \\
8 & 36 & 74.3 & 66.6 & 57.4 & 40.3 & 94.8 \\
9 & 10.8 & 10.3 & 46.5 & 47.9 & 57.5 & 95.2 \\
10 & 17.7 & 93.6 & 35.9 & 58.7 & 45.9 & 12 \\
11 & 38.8 & 97.5 & 55.2 & 41.7 & 60.5 & 82.7 \\
12 & 60.9 & 96.4 & 86 & 28.9 & 93.1 & 72.3 \\
13 & 70.3 & 45.8 & 65.3 & 35.3 & 34.3 & 98.8 \\
14 & 20.5 & 75.6 & 13.1 & 60 & 53.3 & 18.3 \\
15 & 17.9 & 74.8 & 54.2 & 66.7 & 52.1 & 15.8 \\
16 & 51.8 & 19.8 & 52.3 & 74.2 & 73.6 & 84.7 \\
17 & 11.3 & 27.3 & 42.7 & 72.3 & 68.9 & 37.4 \\
18 & 58.7 & 42.1 & 95.9 & 26.6 & 51.6 & 96.4 \\
19 & 41.4 & 51.6 & 83 & 75.4 & 20.5 & 72 \\
20 & 99.7 & 87.1 & 87.5 & 96.9 & 58.6 & 39 \\
21 & 25.6 & 14.6 & 52 & 19.1 & 44.3 & 51.3 \\
22 & 65.1 & 97.3 & 79.4 & 68 & 53.8 & 55.5 \\
23 & 40.4 & 33 & 74.5 & 21.7 & 13.9 & 55.7 \\
24 & 19.4 & 20.1 & 77.5 & 74.1 & 60.9 & 71 \\
25 & 54.2 & 99.3 & 20.8 & 69.9 & 47.8 & 12.2 \\
26 & 80.1 & 27.5 & 51.3 & 95.8 & 21.7 & 12.6 \\
27 & 82.9 & 38.1 & 43.3 & 75.3 & 16.8 & 26.6 \\
28 & 98.6 & 81.8 & 93.8 & 15.9 & 40.3 & 35.8 \\
29 & 77.3 & 40.3 & 95.6 & 52.5 & 96.1 & 44.2 \\
30 & 38.6 & 58.3 & 37.8 & 66.1 & 16 & 69.9 \\
\hline & & & & & &
\end{tabular}

of points $\mathrm{C}$ or B. However, the decision maker may prefer another Pareto optimal point (we have to keep in mind that in the DEA method, each DMU attempts to show itself in the best light, of course within limits of reality and feasibility). The decision maker can choose whether to emphasise the efficiency of the first or the second stage or to find a balance between these. If the decision maker prefers to optimise the efficiency of the first stage, the regions of point $\mathrm{E}$ will be the preferred search direction for the Pareto solution, meaning that (in the case of Example 1) the second stage will be the weak link; an external evaluator may require this to be improved, as it will be at the lowest possible level. The regions of point A will be the same with respect to the second stage.

In the weak link approach, one essential issue is the position of the efficiency frontier with respect to the line $e_{2}=e_{1}$. If the entire efficiency frontier is above this line, the overall efficiency will be always equal to $e_{1}$ (i.e. the first stage will always be the weak link). In this case, the decision maker of the selected DMU may prefer this to be ranked the highest of all the DMUs, and will prefer the region of point E. However, he or she must keep in mind that the second stage will also be examined in a deeper evaluation of this DMU, and in this situation it would be presented in the worst possible light (although its efficiency will be higher than that of the first stage). In the case where the whole efficiency frontier is below the line $e_{2}=e_{1}$, an equivalent consideration will apply to the second stage, which will always be the weak link. 


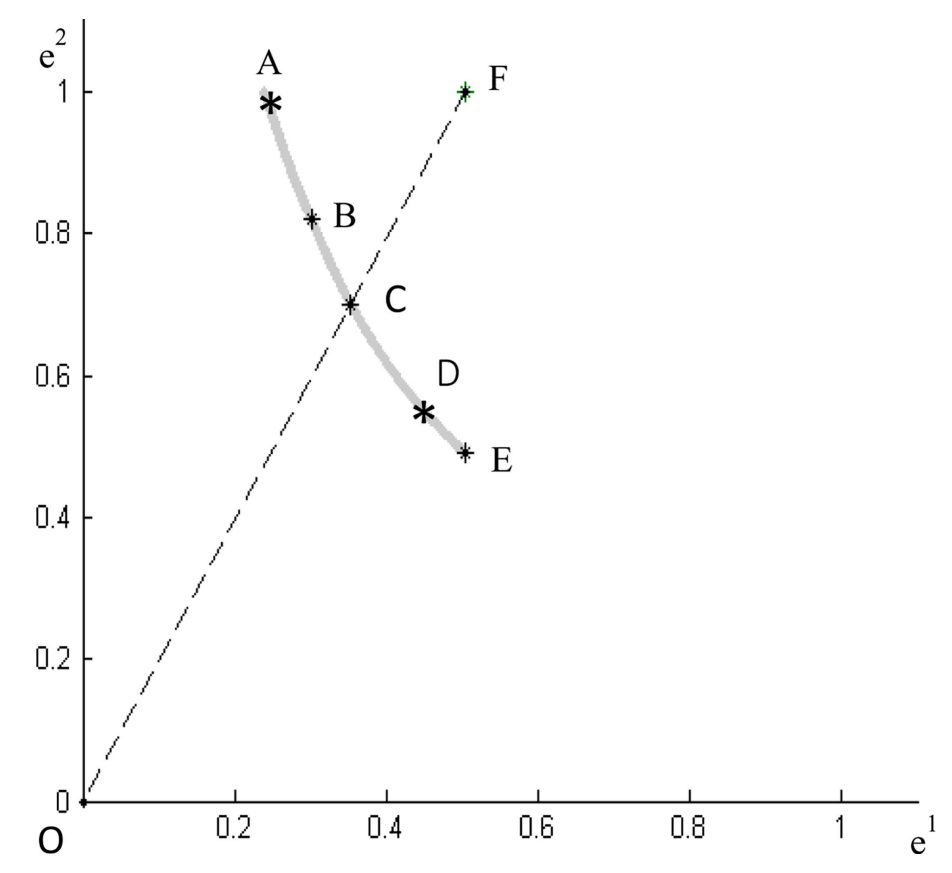

Figure 1. Pareto frontier for Example $1, k_{0}=18$.

In other situations, including that shown in Figure 1, the efficiency frontier crosses the line $e_{2}=e_{1}$. In this case, the decision maker can choose the stage that will be the weak link. Depending on this choice, the regions around A (where the first stage is the weak link and thus deciding about the rating, but evaluated on its lowest possible level, the second stage being evaluated on its highest levels) or E (a similar situation to point A, but relating to the second stage) might be preferred. And always the decision maker has to remember that extreme regions of the efficiency frontier show one of the stages in the best possible and the other stage in the worst possible light. So he or she may prefer to choose a compromise, a point in the interior part of the efficiency frontier, above or below the line $e_{2}=e_{1}$. He or she has to keep in mind that the ranking (thus the weak link) is one thing and a deeper evaluation of both stages is another thing, both being important.

The fuzzy approach proposed in this paper and explained in the next section can help the decision maker to do this.

\section{Aspiration LeVels AND FUZZy BiCRiteria PROGRAMming}

Zimmermann [20] introduced fuzzy sets that can be used to express the decision maker's preferences, for example using aspiration levels. Let $\mathcal{M}=\left(m_{1}, m_{2}\right)$ represent a two-dimensional vector that can take on various values. At the moment of decision making, the decision maker does not know its values, but is able to say which values of the vector would be more satisfactory. We assume that the general preference is for larger values of $m_{1}$ and $m_{2}$, but the definition of "large" recognised by the decision maker is (i) not crisp and (ii) assumes that over a certain level, the numbers are considered to be "large", regardless of the amount by which they exceed this limit. This level is called the aspiration level. In the case of $\left(m_{1}, m_{2}\right)$ considered here, the decision maker can determine the aspiration levels $M_{1}$ and $M_{2}$ for both coordinates and the respective admissible violations $V_{1}$ and $V_{2}$. These values mean that if $m_{1} \geq M_{1}$ (and similarly if $m_{2} \geq M_{2}$ ), the decision maker will be completely satisfied with the value $m_{1}\left(m_{2}\right)$, and if $m_{1} \leq M_{1}-V_{1}$ (similarly, if $m_{2} \leq M_{2}-V_{2}$, the decision maker will be completely dissatisfied). For the other values, there are various possible definitions of the membership functions 


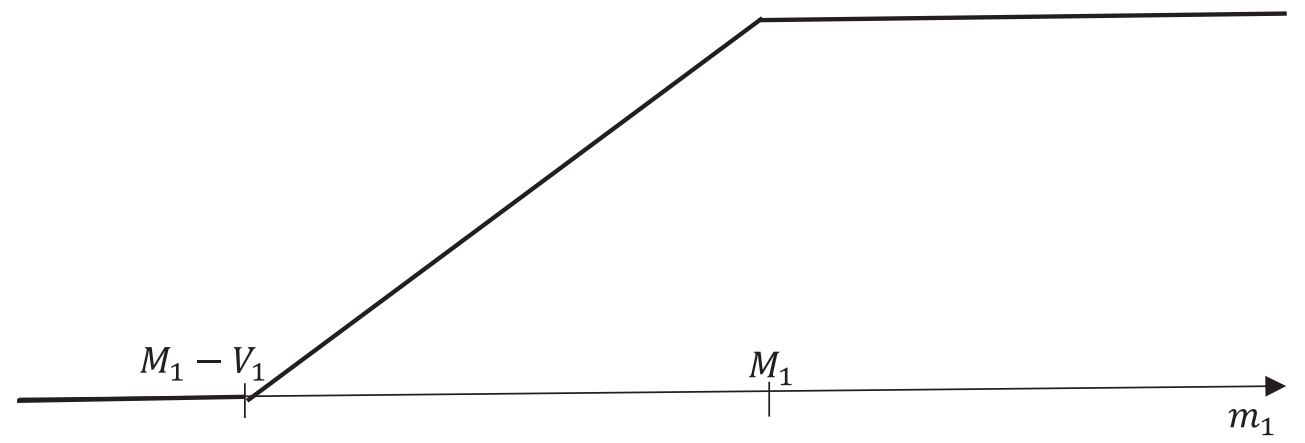

FIGURE 2. The membership function $\mu_{m_{1}}\left(m_{1}\right)(3.1)$.

$\mu_{m_{1}}\left(m_{1}\right)$ and $\mu_{m_{2}}\left(m_{2}\right)$ that represent the degree of satisfaction of the decision maker with the values $m_{1}, m_{2}$. Following Zimmermann [20] we assume that:

$$
\mu_{m_{1}}\left(m_{1}\right)=\left\{\begin{array}{lll}
0 & \text { if } & m_{1} \leq M_{1}-V_{1} \\
\frac{m_{1}}{V_{1}}+1-\frac{M_{1}}{V_{1}} & \text { if } & M_{1}-V_{1}<m_{1} \leq M_{1} . \\
1 & \text { if } & m_{1}>M_{1}
\end{array}\right.
$$

The definition of $\mu_{m_{2}}\left(m_{2}\right)$ is analogous.

Figure 2 illustrates the membership function $\mu_{m_{1}}\left(m_{1}\right)$, which represents the satisfaction of the decision maker with the value $m_{1}$ for which the general goal is maximisation.

The aim of using an aspiration level and admissible violation is to define two threshold values, a lower and an upper value, which define the limits within which the changes of the magnitude in question are of interest to the decision maker. The values of this magnitude do not need to exceed the upper value in order to be to some extent satisfactory; they also cannot fall below the lower limit, since they are then not satisfactory, even if they are close to it. If they exceed the upper limit, then the satisfaction is complete, regardless of how much this value is exceeded by.

We now describe the application of user aspiration levels in bicriterial programming. We will apply this approach to the problem in (2.1), but we first consider the general formulation of bicriteria programming in order to introduce the necessary definitions.

We define a general bicriteria problem for which problem (2.1) is a particular case:

$$
\begin{aligned}
& f_{1}(\boldsymbol{x}) \rightarrow \max \\
& f_{2}(\boldsymbol{x}) \rightarrow \max \\
& \boldsymbol{A}(x)\left(\begin{array}{l}
= \\
\leq \\
\geq
\end{array}\right) \boldsymbol{B}
\end{aligned}
$$

where $\boldsymbol{x}$ is a vector of decision variables, $\boldsymbol{A}$ represents the left-hand side of the constraints and $\boldsymbol{B}$ the right-hand side. Let $\left(Z_{1}, Z_{2}\right)$ denote the ideal point of (3.2); thus, $Z_{i}$ is the optimal value of (3.2) with $f_{i}$ the only objective function in $(3.2)(i=1,2)$.

Both objective functions are maximised. However, we assume that for each objective function there is a certain limit over which the value of the objective function is completely satisfactory to the decision maker (the aspiration level) and that the transition from completely satisfactory values to completely unsatisfactory ones is not crisp (i.e. there is an admissible violation). The aspiration levels and the admissible violations must be defined for both objective functions. Let them be denoted as $M_{1}, V_{1}, M_{2}, V_{2}$, respectively, and let the 
membership functions $\mu_{m_{1}}, \mu_{m_{2}}$ be defined on the domains of the values of $f_{1}$ and $f_{2}$, respectively. We represent the user satisfaction by the values of the individual objective functions.

We then have the following definition:

Definition 3.1 ( $[7,17])$. The fuzzy Pareto solution of (3.2) is a vector $\boldsymbol{x}_{0}$ that fulfils the constraints of (3.2) and for which there does not exist any vector $\boldsymbol{x}_{1}$ fulfilling the constraints of (3.2) such that $\mu_{m_{1}}\left(f_{1}\left(\boldsymbol{x}_{1}\right)\right) \geq$ $\mu_{m_{1}}\left(f_{1}\left(\boldsymbol{x}_{0}\right)\right)$ and $\mu_{m_{2}}\left(f_{2}\left(\boldsymbol{x}_{1}\right)\right) \geq \mu_{m_{2}}\left(f_{2}\left(\boldsymbol{x}_{0}\right)\right)$ and at least one of the following inequalities hold:

(a) $\mu_{m_{1}}\left(f_{1}\left(\boldsymbol{x}_{1}\right)\right)>\mu_{m_{1}}\left(f_{1}\left(\boldsymbol{x}_{0}\right)\right)$

(b) $\mu_{m_{2}}\left(f_{2}\left(\boldsymbol{x}_{1}\right)\right)>\mu_{m_{2}}\left(f_{2}\left(\boldsymbol{x}_{0}\right)\right)$.

The interpretation of the fuzzy Pareto solution of (3.2) is as follows. Fuzzy Pareto solutions are vectors for which no other vectors exist with which the decision maker would be more satisfied, at least according to one criterion. The satisfaction with the other criterion must remain at least at the same level. Thus, the fuzzy Pareto optimal solutions maximise the satisfaction of the decision maker with respect to both of the objective functions that are maximised, while for each objective function, there is a threshold over which the decision maker begins to be somewhat satisfied and another threshold over which he/she is completely satisfied with the value of the corresponding criterion. The set of fuzzy Pareto optimal solutions depends strongly on the decision maker's preferences, expressed by means of fuzzy sets (3.1) and defined on the space of values of both objective functions.

In order to determine a Pareto optimal solution to (3.2), we can start $[7,17]$ by solving the so-called min-max problem:

$$
\begin{aligned}
& \lambda \rightarrow \max \\
& \mu_{m_{1}}\left(f_{1}(\boldsymbol{x})\right) \geq \lambda \\
& \mu_{m_{2}}\left(f_{2}(\boldsymbol{x})\right) \geq \lambda \\
& \boldsymbol{A}(\boldsymbol{x})\left(\begin{array}{l}
= \\
\leq \\
\geq
\end{array}\right) \boldsymbol{B} .
\end{aligned}
$$

It can be observed that problem (3.3) is equivalent to maximising the objective function determined on $\mathcal{R}^{2}$, which defines a fuzzy set on $\mathcal{R}^{2}$, i.e. the universe of the values $\left(f_{1}(\boldsymbol{x}), f_{2}(\boldsymbol{x})\right)$ of $(3.3)$ :

$$
\mu_{\mathcal{M}}\left(e_{1}, e_{2}\right)=\min \left(\mu_{m_{1}}\left(e_{1}\right), \mu_{m_{2}}\left(e_{2}\right)\right)
$$

with the same constraints as in (3.3). Thus, it is a problem of the max-min type.

Problem (3.3) can be reduced to a linear problem if the membership functions are piecewise linear and if problem (3.2) is linear. Since we assume that the membership functions are of type (3.1), this is the case here. The corresponding linear problem is:

$$
\begin{aligned}
& \lambda \rightarrow \max \\
& \frac{f_{1}(\boldsymbol{x})}{V_{1}}+1-\frac{M_{1}}{V_{1}} \geq \lambda \\
& \frac{f_{2}(\boldsymbol{x})}{V_{2}}+1-\frac{M_{2}}{V_{2}} \geq \lambda \\
& A(\boldsymbol{x})\left(\begin{array}{l}
= \\
\leq \\
\geq
\end{array}\right) \boldsymbol{B} .
\end{aligned}
$$

We denote the solution to (3.5) by $\boldsymbol{x}^{*}$. For problem (3.5), we check whether $\boldsymbol{x}^{*}$ is a unique solution (using the standard approach of checking the values of reduced costs via the simplex method or some other method, for 
example the one proposed by Appa [1]). If so, it is the Pareto optimal solution; if not, the following problem must be solved [7]:

$$
\begin{aligned}
& \lambda_{1}+\lambda_{2} \rightarrow \max \\
& \mu_{m_{1}}\left(f_{1}(\boldsymbol{x})\right) \geq \lambda_{1} \geq \mu_{m_{1}}\left(f_{1}\left(\boldsymbol{x}^{*}\right)\right) \\
& \mu_{m_{2}}\left(f_{2}(\boldsymbol{x})\right) \geq \lambda_{2} \geq \mu_{m_{2}}\left(f_{2}\left(\boldsymbol{x}^{*}\right)\right) \\
& \boldsymbol{A}(\boldsymbol{x})\left(\begin{array}{l}
= \\
\leq \\
\geq
\end{array}\right) \boldsymbol{B} .
\end{aligned}
$$

In an analogous way to the previous example, problem (3.6) can be addressed by solving a linear programming problem, if problem (3.2) is linear and the membership functions in (3.1) are assumed. The linear programming problem in this case is as follows:

$$
\begin{aligned}
& \lambda_{1}+\lambda_{2} \rightarrow \max \\
& \frac{f_{1}(\boldsymbol{x})}{V_{1}}+1-\frac{M_{1}}{V_{1}} \geq \lambda_{1} \geq \frac{f_{1}\left(\boldsymbol{x}^{*}\right)}{V_{1}}+1-\frac{M_{1}}{V_{1}} \\
& \frac{f_{2}(\boldsymbol{x})}{V_{2}}+1-\frac{M_{2}}{V_{2}} \geq \lambda_{2} \geq \frac{f_{2}\left(\boldsymbol{x}^{*}\right)}{V_{2}}+1-\frac{M_{2}}{V_{2}} \\
& \boldsymbol{A}(\boldsymbol{x})\left(\begin{array}{l}
= \\
\leq \\
\geq
\end{array}\right) \boldsymbol{B} .
\end{aligned}
$$

The solution to (3.7) will be a fuzzy Pareto optimal solution.

Theorem 3.2. Using formula (3.1) and referring to the ideal point of (3.2), then if we assume $M_{1}=V_{1}=Z_{1}$ and $M_{2}=V_{2}=Z_{2}$, the solution to problem (3.3) will be a Pareto optimal point of (3.2).

Theorem 3.2, which is straightforward to prove, assures us that by making the choice of the fuzzy preferences indicated in the theorem, we choose a Pareto optimal solution. If this is not satisfactory to the decision maker, it can be adjusted by changing the values $M_{1}, V_{1}, M_{2}, V_{2}$, which will be described more in detail later on in the paper.

\section{Applichtion of aspiration levels to DEA}

Here we will apply an approach based on aspiration levels to the two-stage case of DEA, using the weak link approach. As explained above, in this approach, one of the Pareto optimal solutions to problem (2.1) must be chosen. The decision maker must give information as to which point is preferrred. This may be based on the influence of each process, taken independently (problems (2.5) and (2.6)), on the efficiency of the two-stage process.

In the approaches presented in the literature thus far Despotis et al. [4,5], the decision maker has had the choice between approaches (i) and (ii) in Section 2. Approach (i) means that the ratio of the influence degrees of the two stages, taken independently, on the two-stage case is proportional to the ratio of the ideal efficiencies. Approach (ii) means that the point representing both efficiencies in the two-stage case should be as close as possible to the point representing the ideal efficiencies.

The fuzzy approach (based on aspiration levels) allows the decision maker to introduce other requirements. The decision maker may be asked to give the values $M_{1}^{k}, V_{1}^{k}, M_{2}^{k}, V_{2}^{k}$, and $\mu_{1}^{k}\left(f_{1}^{k}(\boldsymbol{x})\right)$ and $\mu_{2}^{k}\left(f_{2}^{k}(\boldsymbol{x})\right)$ can be 
defined as in (3.1). The following problem can then be solved (for $\mathrm{DMU}_{k_{0}}$ ):

$$
\begin{aligned}
& \lambda \rightarrow \max \\
& \mu_{m_{1}}\left(\frac{\sum_{l=1}^{L} t_{l}^{k_{0}} z_{l}^{k_{0}}}{\sum_{i=1}^{I} w_{i}^{k_{0}} x_{i}^{k_{0}}}\right) \geq \lambda \\
& \mu_{m_{2}}\left(\frac{\sum_{j=1}^{J} v_{j}^{k_{0}} y_{j}^{k_{0}}}{\sum_{l=1}^{L} t_{l}^{k_{0}} z_{l}^{k_{0}}}\right) \geq \lambda \\
& \frac{\sum_{l=1}^{L} t_{l}^{k_{0}} z_{l}^{k}}{\sum_{i=1}^{I} w_{i}^{k_{0}} x_{i}^{k}} \leq 1, k \in\{1,2, \ldots K\} \backslash\left\{k_{0}\right\} \\
& \frac{\sum_{j=1}^{J} v_{j}^{k_{0}} y_{j}^{k}}{\sum_{l=1}^{L} t_{l}^{k_{0}} z_{l}^{k}} \leq 1, k \in\{1,2, \ldots K\} \backslash\left\{k_{0}\right\}
\end{aligned}
$$

where $t_{l}^{k_{0}}, v_{j}^{k_{0}}, w_{i}^{k_{0}} \geq 0, i=1, \ldots, I, j=1, \ldots, J, l=1, \ldots, L$ are the decision variables.

With necessary corrections, this problem represents the linearisation and by problems (3.6) and (3.7). A solution $\left(\vartheta_{0}, \xi_{0}\right)$ is obtained, where $\min \left(\vartheta_{0}, \xi_{0}\right)$ is the overall evaluation of $\mathrm{DMU}_{0}$.

The Pareto optimal solution of problem (3.2), with $f_{1}^{k}(\boldsymbol{x})$ and $f_{2}^{k}(\boldsymbol{x})$ equal to the objective functions of (2.1) according to Definition 3.1, can then be found. This can be done solely by means of solving linear programming problems. The problem of choosing a Pareto optimal solution no longer exists since the Pareto solution found will be based on the decision maker's preferences, expressed by means of

$$
\mu_{m_{1}}\left(\frac{\sum_{l=1}^{L} t_{l}^{k_{0}} z_{l}^{k_{0}}}{\sum_{i=1}^{I} w_{i}^{k_{0}} x_{i}^{k_{0}}}\right) \text { and } \mu_{m_{2}}\left(\frac{\sum_{j=1}^{J} v_{j}^{k_{0}} y_{j}^{k_{0}}}{\sum_{l=1}^{L} t_{l}^{k_{0}} z_{l}^{k_{0}}}\right) .
$$

The membership functions (4.1) and (4.2) will be defined as in (3.1). The decision maker may give different values for $M_{1}, M_{2}, V_{1}, V_{2}$; he or she is free to give any values within the intervals $\left[0, T_{k_{0}}\right]$ for $M_{1}$ and $V_{1}$ and $\left[0, P_{k_{0}}\right]$ for $M_{2}$ and $V_{2}$. However, the following interactive procedure is suggested:

(A) Solve problem (4.1), (4.2) for $M_{1}=V_{1}=T_{k_{0}}, M_{2}=V_{2}=P_{k_{0}}$. According to Theorem 3.2, a Pareto optimal solution will be obtained (identical to the one found via the approach with the above mentioned assumption (i)).

(B) If $\mu_{m_{1}}\left(\xi_{0}\right)<1$ and is too low according to the decision maker, and $\mu_{m_{2}}\left(\vartheta_{0}\right)$ is higher than expected, decrease $M_{2}$ and/or $T_{1}$ stepwise (by a small $\varepsilon$ ) or increase $M_{1}$ and/or $T_{2}$, also stepwise, as long as $\mu_{m_{1}}\left(\xi_{0}\right)$ is too low and $\mu_{m_{2}}\left(\vartheta_{0}\right)$ is high enough, and all the parameters remain in the respective intervals $\left(\left[0, T_{k_{0}}\right]\right.$ for $M_{1}$ and $V_{1}$ and $\left[0, P_{k_{0}}\right]$ for $M_{2}$ and $\left.V_{2}\right)$. Stop when $\mu_{m_{1}}\left(\xi_{0}\right)$ is high enough or $\mu_{m_{2}}\left(\vartheta_{0}\right)$ is too low, or when the parameters of the fuzzy numbers can no longer be decreased/increased in the desired direction since they would fall outside these intervals;

(C) If $\mu_{m_{2}}\left(\vartheta_{0}\right)<1$ and is too low according to the decision maker, and $\mu_{m_{1}}\left(\xi_{0}\right)$ is higher than desired, act in an analogous way to the above;

(D) If both $\mu_{m_{1}}\left(\xi_{0}\right)$ and $\mu_{m_{2}}\left(\vartheta_{0}\right)$ are too low, either accept the solution or choose a stage to be "sacrificed", i.e. accepting a still lower satisfactory efficiency for this. It may be necessary to "sacrifice" the stage which is not the weak link, so that the ranking position of the DMU is increased. If second stage is to be sacrificed, decrease $M_{2}$ and/or $T_{1}$ stepwise (by a small $\varepsilon$ ) or increase $M_{1}$ and/or $T_{2}$, also stepwise, as long as $\mu_{m_{1}}\left(\xi_{0}\right)$ is too low and all the parameters remain within the respective intervals $\left(\left[0, T_{k_{0}}\right]\right.$ for $M_{1}$ and $V_{1}$ and $\left[0, P_{k_{0}}\right]$ for $M_{2}$ and $V_{2}$ ). If the first stage is to be sacrificed, act in an analogous way.

(E) If both $\mu_{m_{1}}\left(\xi_{0}\right)$ and $\mu_{m_{2}}\left(\vartheta_{0}\right)$ are at an acceptable level, stop.

The values $M_{1}^{k}, V_{1}^{k}, M_{2}^{k}, V_{2}^{k}$ help the decision maker to express the desired influence degree of the ideal values of (2.1) on the solution to the two-stage case in the weak link approach. For example, if $M_{1}^{k}$ is close to $T_{k}$ and $V_{1}^{k}$ is a small number, we have a fuzzy number similar to the following (Fig. 3). 


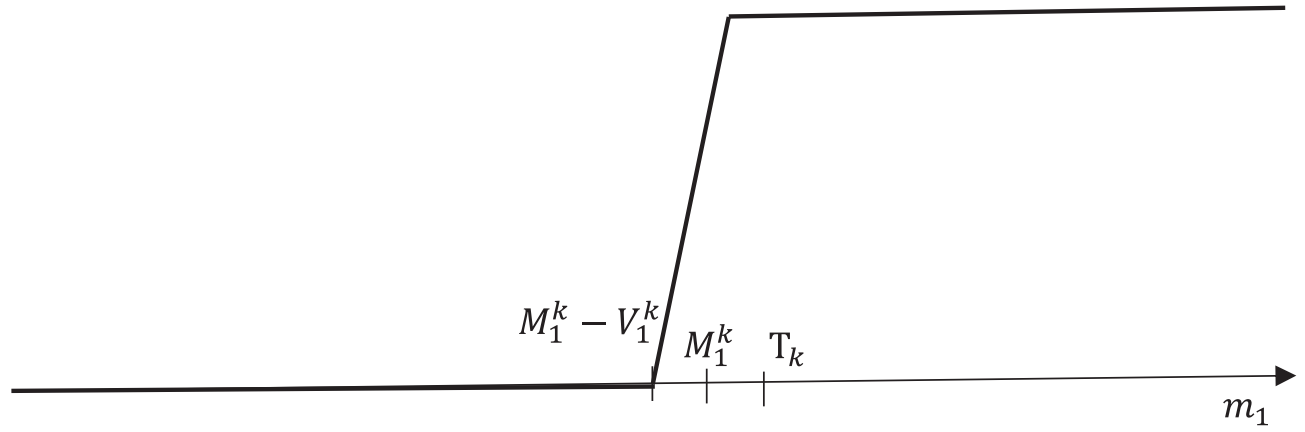

FiguRE 3 . The membership function $\mu_{m_{1}}\left(m_{1}\right)$ for $M_{1}^{k}$ close to $T_{k}$ and for a small value of $V_{1}^{k}$.

In this case, the influence of $T_{k}$ is required to be very strong. Only values close to it have a positive satisfaction degree.

If $M_{1}^{k}$ decreases and/or $V_{1}^{k}$ increases, the influence of $T_{k}$ becomes smaller or is less restrictive. Of course, the relations between $M_{1}^{k}, V_{1}^{k}$ and $M_{2}^{k}, V_{2}^{k}$ are important. For example, if we have $M_{1}^{k}=V_{1}^{k}=T_{k}$ and $M_{2}^{k}=V_{2}^{k}=$ $P_{\mathrm{k}}$, the influence of $T_{k}$ and $P_{\mathrm{k}}$ is proportional to the ratio $T_{k} / P_{k}$, which gives us case (i) from Section 2.

The proposal is illustrated by means of Example 2 .

\section{Example 2}

We take the data from Example 1 and the following functions of type (3.1):

(a) For $k_{o}=18, M_{1}^{18}=T_{18}, V_{1}^{18}=T_{18}, M_{2}^{18}=P_{18}, V_{2}^{k}=P_{18}$ (maximal satisfaction is attained at the ideal point and is zero if one of the criteria takes on a value of zero).

The solution to problem (2.1) according to Definition 3.1 is the point C in Figure 1, and thus we obtain exactly the same solution as in Example 1, approach (i) (step A in the procedure).

Function (3.4) is then as follows:

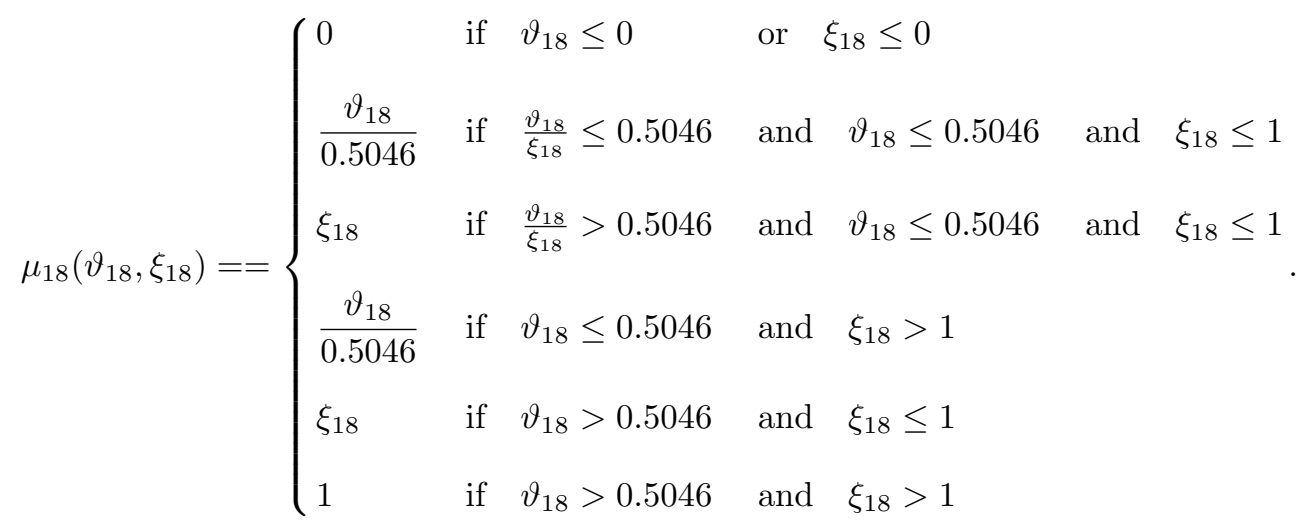

(b) For $k_{o}=18, M_{1}^{18}=T_{18}, V_{1}^{18}=0.25 T_{18}, M_{2}^{18}=0.75 P_{18}, V_{2}^{18}=0.75 P_{18}$. For the first objective function, the satisfaction is positive only for values between $0.75 T_{18}$ and $T_{18}$ (the decision maker requires a strong influence from the first stage). For the second objective function, the decision maker is already completely satisfied with the value of $0.75 P_{18}$, and thus requires a lower influence with respect to the second stage. This solution might be derived from the solution obtained in a) via step B (decreasing $V_{1}^{18}$ and $M_{2}^{18}$ in 


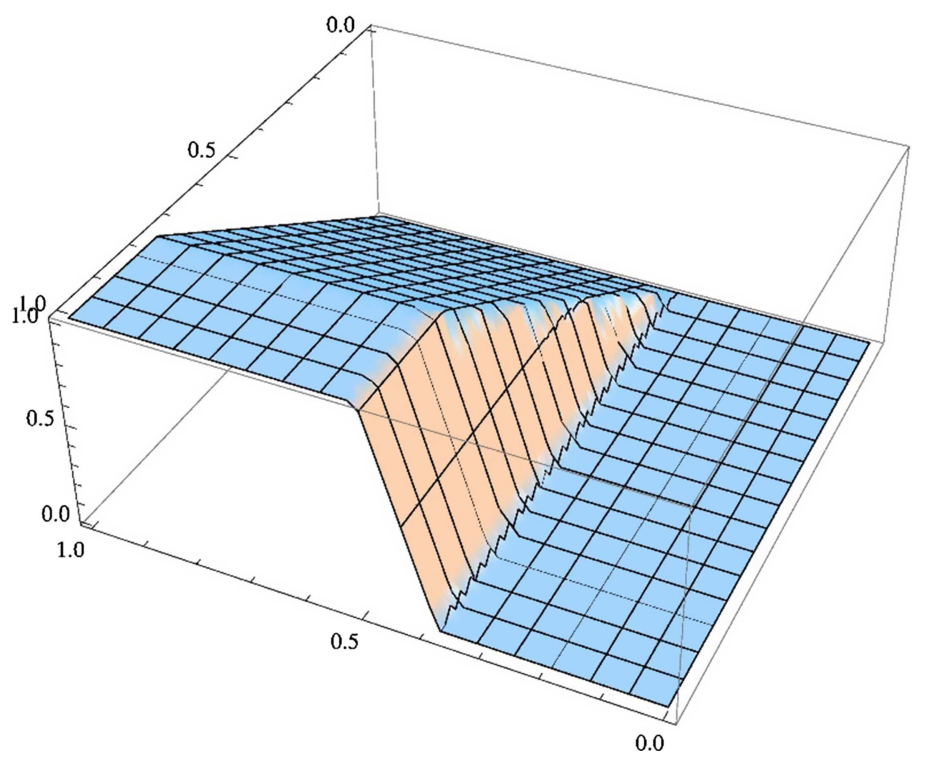

Figure 4. Function (3.4) for Example 2b.

order to increase $\mu_{m_{1}}\left(\frac{\sum_{l=1}^{L} t_{l}^{k_{0}} z_{l}^{k_{0}}}{\sum_{i=1}^{I} w_{i}^{k_{0}} x_{i}^{k_{0}}}\right)$, at the cost of $\left.\mu_{m_{2}}\left(\frac{\sum_{j=1}^{J} v_{j}^{k_{0}} y_{j}^{k_{0}}}{\sum_{l=1}^{L} t_{l}^{k_{0}} z_{l}^{k_{0}}}\right)\right)$, and then step C (decreasing $V_{1}^{18}$ in order to correct the previous step, i.e. increasing $\mu_{m_{2}}\left(\frac{\sum_{j=1}^{J} v_{j}^{k_{0}} y_{j}^{k_{0}}}{\sum_{l=1}^{L} t_{l}^{k_{0}} z_{l}^{k_{0}}}\right)$ at the cost of $\left.\mu_{m_{1}}\left(\frac{\sum_{l=1}^{L} t_{l}^{k_{0}} z_{l}^{k_{0}}}{\sum_{i=1}^{I} w_{i}^{k_{0}} x_{i}^{k_{0}}}\right)\right)$.

Function (3.4) is then as follows:

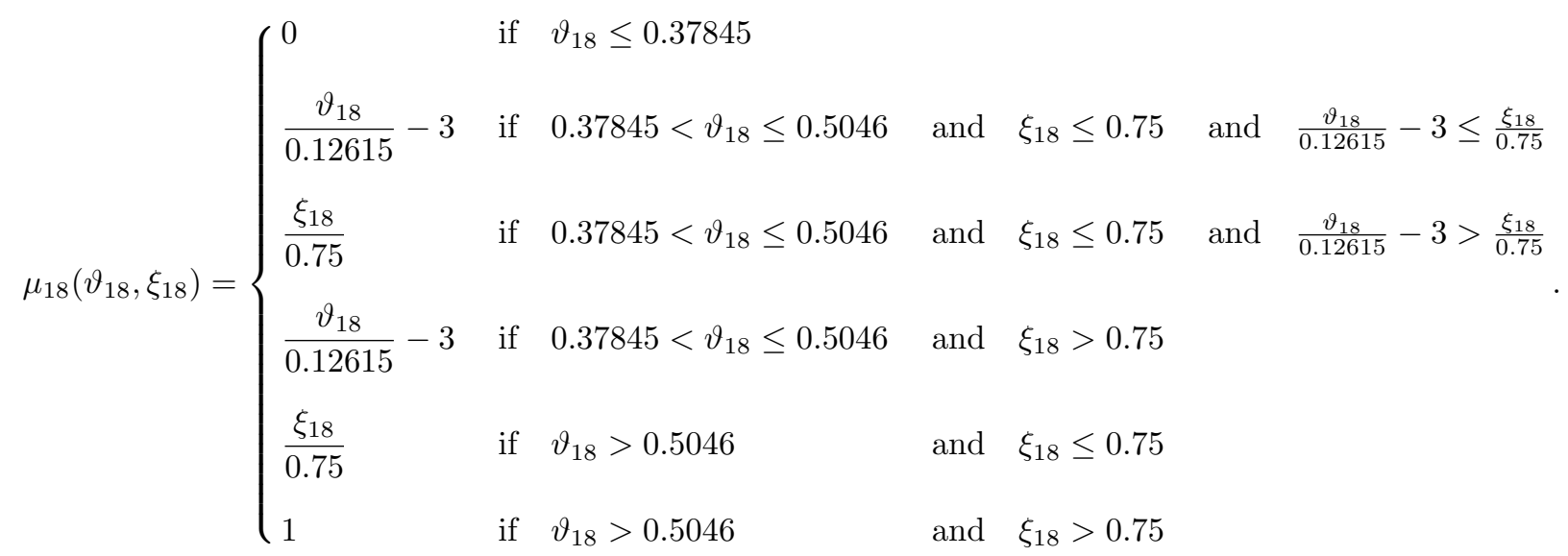

The solution to problem (2.1) according to Definition 3.1 for Example $2 \mathrm{~b}$ would be the point $D=$ $(0.468,0.528)$, located between $C$ and $E$ in Figure 1. Thus we have $e_{18}=0.468$ (Fig 4).

The following theorem is then true:

Theorem 4.1. For each point $X=\left(x_{1}, x_{2}\right)$ on the crisp Pareto frontier of problem (2.1), there exist $M_{1}^{k}, \quad V_{1}^{k}, M_{2}^{k}, V_{2}^{k}$ and functions (3.1) such that this point is the unique fuzzy Pareto solution to problem (2.1) according to Definition 3.1, and the unique solution of problem (3.3). 
Proof. If we take any $0 \leq \lambda \leq 1$ and values of $M_{1}^{k}, \quad V_{1}^{k}, M_{2}^{k}, V_{2}^{k}$ such that $\frac{x_{1}}{V_{1}}+1-\frac{M_{1}}{V_{1}}=\lambda$ and $\frac{x_{2}}{V_{2}}+1-\frac{M_{2}}{V_{2}}=\lambda$, then we have $\mu_{m_{1}}\left(x_{1}\right)=\mu_{m_{2}}\left(x_{2}\right)=\lambda$. Since $X=\left(x_{1}, x_{2}\right)$ is a crisp Pareto optimal solution, $\left(x_{1}, x_{2}\right)$ is a unique solution of (3.3) and a unique fuzzy Pareto optimal solution.

Theorem 4.1 means that for each crisp Pareto optimal solution to (2.1), there exists a family of expert opinions expressed by (3.1) (and indexed by $\lambda$ ) for which this crisp Pareto optimal solution is the only crisp Pareto optimal solution satisfying the expert's opinion on the influence of the ideal point $\left(T_{k_{0}}, P_{k_{0}}\right)$ on the solution to (2.1) according to the weak link approach. This means that each crisp Pareto optimal solution has a chance to be the only solution candidate for (2.1). This resolves the problem of the lack of uniqueness of the solution of (2.1), as long as the experts are able to decide which element is to be emphasised and to what extent with respect to the other elements: the ranking position of the DMU, the efficiency of the first stage or the efficiency of the second stage.

As for the practical question of solving the relevant problems, problems (3.2), (3.3) and (3.5) are nonlinear for the weak link approach to DEA, since here we are using the nonlinear objective functions of (2.1). However, they can first be reduced to linear problems via a $\mathrm{C}-\mathrm{C}$ transformation and then by a bisection search as in [4].

\section{Conclusions}

This paper proposes the application of fuzzy preferences and fuzzy bicriterial programming in the weak link approach to the two-stage DEA. The proposed method helps in identifying the final solution in the weak link approach, which in the original weak link approach is not unequivocal. An indication of the search direction at the Pareto frontier is required, and the proposed fuzzy approach may be helpful here when fuzzy numbers are used to express the decision maker's preferences.

The fuzzy approach does not automatically resolve the problem of the equivocality of the solution in the weak link approach. In an extreme case, it may happen that in the beginning it will not bring anything, since the decision maker will not be able to express the relevant preferences adequately. However, the possibility then arises of making these requirements more precise (the procedure for this is outlined in this paper) and the final solution will be determined in a stepwise manner. The important theorem is proven in the paper that for each candidate solution (i.e. each Pareto solution) in the original weak link problem, there exists a family of expert opinions for which the candidate solution will be the only solution satisfying the decision maker. Thus, each Pareto optimal solution has a chance to become the solution selected according to the decision maker's preferences.

Future research is expected to involve:

- A more general model of the two-stage and multi-stage DEA (e.g. allowing for additional inputs in the second and later stages);

- Interactive determination of the fuzzy sets, to allow them to exactly express the decision maker's preferences;

- Real-world applications of the proposed method.

The proposed approach (fuzzy numbers as a way of expressing the decision maker's preferences) can also be applied to decomposition approaches. Fuzzy versions of the decomposition approaches in the literature use fuzzy numbers as an expression of inexactly known values rather than an expression of the decision maker's preferences.

\section{REFERENCES}

[1] G. Appa, On the uniqueness of solutions to linear programs. J. Oper. Res. Soc. 53 (2002) 1127-1132.

[2] Y. Chen, W.D. Cook, N. Li and J. Zhu, Additive efficiency decomposition in two-stage DEA. Eur. J. Oper. Res. 196 (2009) 1170-1176.

[3] D.K. Despotis, G. Koronakos and D. Sotiros, A multi-objective programming approach to network DEA with an application to the assessment of the academic research activity. Proc. Comput. Sci. 55 (2015) 370-379.

[4] D.K. Despotis, G. Koronakos and D. Sotiros, The "weak-link" approach to network DEA for two-stage processes. Eur. J. Oper. Res. 254 (2016) 481-492. 
[5] D.K. Despotis, D. Sotiros and G. Koronakos, A network DEA approach for series multi-stage processes. Omega 61 (2016) $35-48$.

[6] A. Emrouznejad, M. Tavana and A. Hatami-Marbini, The state of the art in fuzzy data envelopment analysis. Performance Measurement with Fuzzy Data Envelopment Analysis, in: Vol. 309 of Studies in Fuzziness and Soft Computing. Springer-Verlag (2014), 48.

[7] M. Jiménez and A. Bilbao, Pareto-optimal solutions in fuzzy multi-objective linear programming. Fuzzy Sets Syst. 160 (2009) $2714-2721$.

[8] C. Kao, Efficiency decomposition for general multi-stage systems in data envelopment analysis. Eur. J. Oper. Res. 232 (2014) $117-124$.

[9] S. Lim and J. Zhu, A note on two-stage network DEA model: Frontier projection and duality. Eur. J. Oper. Res. 248 (2016) $342-346$.

[10] R.K. Matin and M.I. Ghahfarokhi, A two-phase modified slack-based measure approach for efficiency measurement and target setting in data envelopment analysis with negative data. IMA J. Manage. Math. 26 (2015) 83-88.

[11] H. Omrani, K. Shafaat and E. Emrouznejad, An integrated fuzzy clustering cooperative game data envelopment analysis model with application in hospital efficiency. Expert Syst. App. 114 (2018) 615-628.

[12] J. Puri and S.P. Yadav, A fuzzy DEA model with undesirable fuzzy outputs and its application to the banking sector in India. Expert Syst. App. 41 (2014) 6419-6432.

[13] R.K. Shiraz, V. Charles and L. Jalalzadeh, Fuzzy rough DEA model: a possibility and expected value approaches. Expert Syst. App. 41 (2014) 434-444.

[14] M. Tavana, R.K. Shiraz, A. Hatami-Marbini, P.J. Agrell and K. Paryab, Chance-constrained DEA models with random fuzzy inputs and outputs. Knowl.-Based Syst. 52 (2013) 32-52.

[15] W. Wang, W. Lu and P. Liu, A fuzzy multi-objective two-stage DEA model for evaluating the performance of US bank holding companies. Expert Syst. App. 41 (2014) 4290-4297.

[16] K.J. Watson, J.H. Blackstone and S.C. Gardiner, The evolution of a management philosophy: The theory of constraints. J. Oper. Manage. 25 (2007) 387-402.

[17] Y. Wu, C. Liu and Y. Lur, Pareto-optimal solution for multiple objective linear programming problems with fuzzy goals. Fuzzy Optim. Decis. Making 14 (2015) 43-55.

[18] A.L. Zerafat, A. Emrouznejad and A. Mustafa, Fuzzy data envelopment analysis: a discrete approach. Expert Syst. App. 39 (2012) 2263-2269.

[19] Z. Zhou, L. Zhao, S. Lui and C. Ma, A generalized fuzzy DEA/AR performance assessment model. Math. Comput. Model. 55 (2012) 2117-2128.

[20] H. Zimmermann, Applications of fuzzy set theory to mathematical programming. Inf. Sci. 36 (1985) $29-58$. 